Methods 105 consecutive patients were enrolled into the study. Each patient was assigned to either insulin or glibenclamide group according to which treatment he accepted outside hospital regularly. Collected the basical clinical informations of all patients. One month after PCI all patients took SPECT to evaluate the condition of myocardial perfusion, and ERNA for LVEF.

Results The ratio of the patients with <TIMI III grade and the incidence of arrhythmia in the three groups were statistically significant difference. In the rest state, the ratio of abnormal myocardial perfusion segments in the three groups were statistically significant difference. The ischaemic myocardial area score group A was significantly higher than group B and group C, group B and group $C$ were no significant difference. ERNA showed that LVEF in the three groups were statistically significant difference. Group A was significantly lower than group $\mathrm{B}$, there were no significant difference between group $\mathrm{B}$ and group $\mathrm{C}$, group $\mathrm{A}$, group $\mathrm{C}$ differences were statistically significant illustrated the LVEF of group A was significantly lower than group B and group C.

Conclusion Glibenclamide would increase myocardial ischaemic area in patients with AMland type 2 diabetes mellitus. Glibenclamide increases the possibility of malignant arrhythmias in the patients with acute myocardial infarction and type 2 diabetes mellitus.

\section{e0411 EFFECTS OF SLEEP APNEA SYNDROME ON MYOCARDIAL ISCHAEMIA IN PATIENTS WITH CORONARY HEART DISEASE DURING NIGHT}

doi:10.1136/hrt.2010.208967.411

Fu Xianghua, Pang Jiangna, Wang Xuechao, Wang Yanbo, Jiang Yunfa, Wu Weili, Hao Guozhen, Gu Xinshun. The 2nd Hospital of Hebei Medical University, Shijiazhuang, Hebei, China

Objective 1. To investigate the relationship between sleep apnea syndrome (SAS) and myocadial ischaemic events in patients with coronary heart disease (CHD). 2. To compare the differences of age, gender distribution, coronary angiography results in CHD patients with and without SAS. 3. To compare the differences of C-reactive protein (CRP) and haemoglobin levels in CHD patients with and without SAS.

Methods $25 \mathrm{CHD}$ patients with typical symptoms of angina and ECG changes were enrolled in this study. After overnight polysomnography (PSG), all the cases were monitored by portable device at night for 7 days in order to exclude the conditions that the cases did not sleep or had waked, apnea and hypopnoea events were recorded during 24: 00-4:00. Blood samples were collected 5-10 min after monitoring, and the levels of haemoglobin and C-reactive protein were examined.

Results 1 . The incidence of myocardial ischaemia caused by apnea and low ventilation was significantly higher in CHD patients with SAS. 2. There were significant differences between the two groups in the decrease of oxygen desaturation and the increase of heart rate. 3. BMI in CHD patients was significantly higher in those with SAS. There were more multi-vessel lesions and long lesions in CHD patients with SAS $(\mathrm{p}<0.05)$. The level of haemoglobin and C-reactive protein were much higher in CHD patients with SAS.

Conclusion 1. The incidence of SAS is much higher in patients with $\mathrm{CHD}$, and the incidence of myocardial ischaemic events is higher in CHD patients with SAS. and the more seriuos respiratory disorders, the more easily myocardial ischaemia happens. With apnea related to myocardial ischaemia and oxygen reduction, has nothing to do with the heart rate. 2. Lesions of SAS in patients with coronary heart disease are heavier than Simple CHD group in coronary angiography. BMI of SAS in patients with coronary heart disease are high than Simple CHD group. 3. The levels of CRP and haemoglobin are higher in CHD patients with SAS.

\section{e0412 OBSTRUCTIVE SLEEP APNEA SYNDROME IS ASSOCIATED WITH INCREASED RISK OF LOW-ANTIPLATELET RESPONSE OF CLOPIDOGREL IN PATIENTS WITH UNSTABLE ANGINA}

doi:10.1136/hrt.2010.208967.412

Fu Xianghua, Li Shiqiang, Wang Qian, Gu Xinshun, Wang Yanbo, Wang Xuechao, Wu Weili, Xue Ling. The 2nd Hospital of Hebei Medical University, Shijiazhuang, Hebei, China

Objective To address the relationship between low antiplatelet response of clopidogrel and Obstructive Sleep Apnea Syndrome (OSAS) in patients with unstable angina pectoris.

Methods Total of 112 patients hospitalised with unstable angina pectoris from February 2008 to December 2009 were enrolled in this randomised consecutive study. All patients accepted routine treatment including clopidogrel, aspirin, low molecular weight heparin daily. Platelet aggregation (PAR) parameters were measured on samples obtained at baseline and $2^{\text {nd }}, 4^{\text {th }}, 6^{\text {th }}$ day. All patients were examined for the presence of sleep-disordered breath into 4 quartiles by ApneaLink. The concentration serum adrenaline and norepinephrine were measured in the morning at 6 a.m. after the sleep study.

Result There were no significant differences in the baseline data in all 4 quartiles. However, there was a significant differences in the number of diabetes patients in the first quartibesity, heale $(p=0.0038)$ compared with other quartiles. At day 2 PAR were inhibited to $63.91 \%$ of baseline $(p<0.01)$ and $88.38 \%(p>0.05)$ of baselinctively, in the first quartile. At each of these time points, platelet activity was significantly higher than in patients in other quartiles. At day 6 platelet aggregation were reduced to $32.37 \%$, and $29.75 \%$ of baseline respectively in group 2 through 4 ( $p<0.01$ for all). PAR was reduced significantly in patients in the second through fourth quartiles at day 6 , but, it showed a lower reduction in the first quartile ( $p>0.05)$. Compared with that in thebidity of OSAS in the second and third were $25.0 \%$ and $14.3 \%(p<0.05)$, only $3.6 \%$ in the fourth group $(p<0.01)$. Meanwhile, the concentratio first group $(60.7 \%)$, the mor $n$ of serum adrenaline and nine were higher in the first quartile than others $(p<0.05)$.

Conclusion OSAS is aicator of low clopidogrel response in unstable angina patients, and higher concentration of epinephrine and norepinephrine in OSAS pa reliable ind tients plaorepinephry a more important role in this situation.

\section{e0413 THE ADVERSE EFFECTS OF GLIBENCLAMIDE ON MYOCARDIA PERFUSION IN PATIENTS WITH ACUTE MYOCARDIAL INFARCTION AND TYPE 2 DIABETES MELLITUS}

doi:10.1136/hrt.2010.208967.413

Fu Xianghua, Wu Weili, Yan Yangmei, Wang Xuechao, Wang Yanbo, Fan Weize, Jiang Yunfa, Hao Guozhen. The Second Hospital of Hebei Medical University

Objective To assess the adverse effects of glibenclamide on the myocardium, for investigating more effective and rational therapy. Methods 115 consecutive patients were enrolled into the study. All patients had clinical histories of acute myocardial infarction and losed the chance of thrombolysis and emergency PCI; Each patient was assigned to either insulin or glibenclamide group according to which treatment he accepted outside hospital regularly. The patients who took glibenclamide for group A, insulin for group B and diet for group $\mathrm{C}$. Collected the basical clinical informations of all patients. One month after PCI all patients took SPECT to evaluate the condition of myocardial perfusion, and ERNA for LVEF.

Results The ratio of the patients with <TIMI III grade in the three groups were statistically significant difference. The incidence of 
arrhythmia of the three groups were significantly difference. In the rest state, the ratio of abnormal myocardial perfusion segments in the three groups were statistically significant difference. The ischaemic myocardial area score group A was significantly higher than group $B$ and group $C$, group $B$ and group $C$ were no significant difference. ERNA showed that LVEF in the three groups were statistically significant difference. Group A was significantly lower than group $B$, there were no significant difference between group $B$ and group $\mathrm{C}$, group $\mathrm{A}$, group $\mathrm{C}$ differences were statistically significant illustrated the LVEF of group A was significantly lower than group B and group C.

Conclusion Glibenclamide would increase myocardial ischaemic area in patients with acute myocardial infarction and type 2 diabetes mellitus. Glibenclamide increases the possibility of malignant arrhythmias in the patients with acute myocardial infarction and type 2 diabetes mellitus.

\section{e0414 LIPID LEVEL OF PATIENTS WITH ACUTE MYOCARDIAL INFARCTION UNDERGOING PRIMARY ANGIOPLASTY IS RELATED WITH PROGNOSIS}

doi:10.1136/hrt.2010.208967.414

Liu Ran, Yan Hongbing. Beijing Anzhen Hospital

Objective TO evaluate the correlation between acute lipids level and on-statins treatment lipids and 1 year major adverse cardiovascular and cerebrovascular events (MACCE), including cardiac death, ischaemic stroke, and recurrent myocardial infarction in patients with ST-elevation myocardial infarction (STEMI) undergoing primary percutaneous coronary intervention (PCI).

Methods Based on the LDL-C level within $24 \mathrm{~h}$ after admission, consecutive 624 patients with STEMI undergoing primary PCI were classified into the normal range of LDL-C $<3.37 \mathrm{mmol} \cdot 1^{-1}(\mathrm{n}=380)$, the critical range of LDL-C $3.37 \sim 4.14 \mathrm{mmol} \cdot \mathrm{l}^{-1}(\mathrm{n}=159)$ or the elevated range of LDL-C $>4.14 \mathrm{mmol} \cdot 1^{-1} \quad(n=85)$. Of these 624 patients, serum lipids levels of 335 patients after statins treatment for 4 weeks underwent follow-up in outpatient settings. Based on the results of follow-up, these 335 patients were divided into the targeted group (LDL-C $<1.81 \mathrm{mmol} \cdot \mathrm{I}^{-1}, \mathrm{n}=77$ ) or the non-targeted group (LDL-C $\geq 1.81 \mathrm{mmol} \cdot \mathrm{l}^{-1}, \mathrm{n}=258$ ). The end point was 1 year MACCE.

Results When compared between different lipid ranges (the normal, the critical and the elevated) of patients, the acute lipid level was not associated with the end points of cardiac death, ischaemic stroke, recurrent myocardial infarction, and MACCE ( $p=0.871$, $0.568,0.978,0.587$ ). After 4 weeks statins treatment, the LDL-C level achieved the goal in 77 patients $(23.0 \%)$. The primary end point occurred in $1.3 \%$ of patients treated with LDL-C $<1.81$ $\mathrm{mmol} \cdot \mathrm{1}^{-1}$ group and in $7.4 \%$ in LDL-C $\geq 1.81 \mathrm{mmol} \cdot \mathrm{1}^{-1}$ group $(p=0.034)$. Logistic regression analysis showed a significant relationship between acute triglyceride (TG) level and ischaemic stroke (OR, 1.226; 95\% CI 1.068 to 1.407; $p=0.004$ ), and between LDL-C level after stain therapy and MACCE (OR, 1.788; 95\% CI 1.091 to 5.233; $\mathrm{p}=0.039$ ). Smoking history (OR, 0.136; 95\% CI 0.016 to $1.115 ; \mathrm{p}=0.036)$ and higher SYNTAX score (OR, 1.544; 95\% CI 5.387 to $33.522 ; \mathrm{p}=0.018$ ) were predictors of 1 year MACCE in the patients with STEMI undergoing primary PCI.

Conclusions 1 year MACCE follow-up investigation in the patients with STEMI undergoing primary PCI suggested high on-treatment LDL-C level was a high risk of increased MACCE although acute lipid level was not associated with MACCE. This finding supports the concept that achieving low LDL-C levels is an important therapeutic parameter in statins-treated patients following STEMI and PCI.

\section{e0415 THE CONTRAST RESEARCH IN SAFETY AND EFFICACY BETWEEN NADROPARIN AND DALTEPARIN BEFORE ELECTIVE PERCUTANEOUS CORONARY INTERVENTION}

doi:10.1136/hrt.2010.208967.415

Liu Ruifang, Yan Hongbing. Beijing Anzhen Hospital

Background To evaluate the contrast research in safety and effective time between nadroparin and dalteparin before elective percutaneous coronary intervention (PCI) in Chinese population. Investigating the relationship among anti-Xa activities APTT and ACT, and if it help to use nadroparin and dalteparin.

Methods A total of 101 consecutive patients undergoing elective PCI were randomised to either nadroparin (50 cases) or dalteparin (51 cases) group for procedural anticoagulation. According to description, the patients in nadroparin or dalteparin group were given nadroparin $(0.01 \mathrm{ml} / \mathrm{kg})$ or dalteparin $(120 \mathrm{IU} / \mathrm{kg})$ subcutaneously more than $48 \mathrm{~h}$ twice per day before PCI. Serial plasma anti-Xa activities ACT and APTT were measured before and after nadroparin and dalteparin were injected. Then coronary angiography (CAG) and PCI was performed. Bleeding complications were classified according to Thrombolysis in Myocardial Infarction (TIMI) criteria. All patients were monitored for anticoagulation before and after $\mathrm{PCI}$ and adverse events (ie, death, myocardial infarction, demands for revascularization) 30 days after PCI.

Results 101 patients were completed. 1. The proportion, plasma anti-Xa activities of patients in nadroparin or dalteparin group above $0.5 \mathrm{IU} / \mathrm{ml}$, exceeded $92 \%$ after $4 \mathrm{~h}$ subcutaneous injection. The proportion descended to $74 \%$ and $36 \%$ in nadroparin group after $8 \mathrm{~h}$ and $12 \mathrm{~h}, 54.9 \%$ and $23.5 \%$ in dalteparin group, which descend more, but had no significantly difference compared with nadroparin group. After $24 \mathrm{~h}$ and $48 \mathrm{~h}$, the proportion of plasma anti-Xa activities of patients in both groups are above $0.5 \mathrm{IU} / \mathrm{ml}$, achieved steady state and exceeded 96.0\%. 2. The plasma anti-Xa activities correlated with APTT significantly in both groups after $4 \mathrm{~h} 24 \mathrm{~h}$ and $48 \mathrm{~h}(\mathrm{p}<0.01)$. The plasma anti-Xa activities hardly correlated with ACT, and have no difference in Statistics (nadroparin group $p=0.075$, dalteparin group $p=0.093$ ). 3 . There were no adverse events in 30 days, and no significantly difference about bleeding events (6.0\% vs $3.9 \%$, p>0.05) between the two groups.

Conclusion The subcutaneous injection of nadroparin or dalteparin for anticoagulation in patients before elective PCI is safe and effective. Anticoagulation effects can be maintained for at least $8 \mathrm{~h}$. APTT can response to the anticoagulation effects of nadroparin or dalteparin by subcutaneous injection. ACT cannot response to the effective anticoagulation effects of nadroparin or dalteparin by subcutaneous injection.

\section{e0416 EFFECT OF CARDIOVASCULAR RISK FACTORS ON SECONDARY PERCUTANEOUS CORONARY REVASCULARIZATION}

doi:10.1136/hrt.2010.208967.416

Liu Chen, Yan Hongbing. Beïing Anzhen Hospital

Objectives An increasing number of patients undergoing percutaneous coronary intervention (PCI) have experienced previous revascularization procedures. However risk factors associated with secondary percutaneous coronary revascularization and their effect sizes were lack of research. The purpose of this large scale crosssectional survey was to investigate quantitative effect of cardiovascular risk factors.

Methods Patients with coronary heart disease hospitalised in the Department of Cardiology of Beijing Anzhen Hospital whose disease was identified by angiography were consecutively enrolled 